Pacific

Journal of

Mathematics

\title{
REAL CLOSED SEPARATION THEOREMS AND APPLICATIONS TO GROUP ALGEBRAS
}

Tim NeTZER AND ANDREAS ThOM 


\title{
REAL CLOSED SEPARATION THEOREMS AND APPLICATIONS TO GROUP ALGEBRAS
}

\author{
Tim NetZer AND ANDREAS Thom
}

Dedicated to Konrad Schmüdgen on the occasion of his 65th birthday

\begin{abstract}
In this paper we prove a strong Hahn-Banach theorem: separation of disjoint convex sets by linear forms is possible without any further conditions if the target field $\mathbb{R}$ is replaced by a more general real closed extension field. From this we deduce a general Positivstellensatz for $*$-algebras, involving representations over real closed fields. We investigate the class of group algebras in more detail. We show that the cone of sums of squares in the augmentation ideal has an interior point if and only if the first cohomology vanishes. For groups with Kazhdan's property (T), the result can be strengthened to interior points in the $\ell^{1}$-metric. We finally reprove some strong Positivstellensätze by Helton and Schmüdgen, using our separation method.
\end{abstract}

\section{Introduction}

In this article we combine techniques from real algebraic geometry, convex geometry, and the unitary representation theory of discrete groups to address various problems that arise in the emerging field of noncommutative real algebraic geometry [Schmüdgen 2009]. Classical results - like Artin's solution of Hilbert's 17th problem strive for a characterization of natural notions of positivity in terms of algebraic certificates. For example, Artin proved that every polynomial in $n$ variables that is positive at every point on $\mathbb{R}^{n}$ must be a sum of squares of rational functions. Much later, Schmüdgen [1991] proved that a strictly positive polynomial on a compact semialgebraic set must be a sum of squares of polynomials plus defining inequalities. More recently, similar questions were asked in a noncommutative context. Typically, the setup involves a $*$-algebra $A$ and a family of representations $\mathscr{F}$. The question is now: Is every self-adjoint element of $A$ that is positive (semi)definite in every representation in $\mathscr{F}_{F}$ necessarily of the form $\sum_{i} a_{i}^{*} a_{i}$ for some $a_{i} \in A$ ? It turned out - similar to the more classical commutative case - that the cone $\Sigma^{2} A=\left\{\sum_{i} a_{i}^{*} a_{i} \mid a_{i} \in A\right\} \subset A$ is an interesting object of study in itself. Natural

Keywords: real closed fields, group rings, Kazhdan's property (T), sums of squares. 
questions are: Is $\Sigma^{2}(A) \cap\left(-\Sigma^{2} A\right)=\{0\}$ ? Is $\Sigma^{2} A$ closed in a natural topology? Does it contain interior points, in the finest locally convex topology, say?

A point $q$ is called an algebraic interior point of a cone $C$ if the cone intersects each line through $q$ in an open interval around $q$. A point is an algebraic interior point if and only if it is an interior point in the finest locally convex topology (see [Cimprič et al. 2011, Proposition 1.3], for example). The question for interior points of cones has the following motivation. If a cone $C$ has an (algebraic) interior point $q$, then for every point $a \in C^{\vee \vee}$ from the double dual, one has $a+\epsilon q \in C$ for all $\epsilon>0$ (see [loc. cit.] for a proof of this well known fact). Using the standard Gelfand-Naimark-Segal construction, this yields the following Positivstellensatz for unital $*$-algebras:

Theorem. Assume that $q$ is an interior point of the cone $\Sigma^{2} A$. If $a=a^{*} \in A$ is positive semidefinite in each $*$-representation of $A$, then $a+\epsilon q \in \Sigma^{2} A$ for all $\epsilon>0$.

Our first main result is a different Positivstellensatz (Theorem 3.12): we prove that each element from a real reduced unital $*$-algebra that is positive in every generalized representation is necessarily in $\Sigma^{2} A$. The notion of a generalized representation involves an extension of the standard real and complex numbers to more general real- and algebraically closed fields.

A natural and vast class of examples of $*$-algebras is given by complex group algebras $\mathbb{C}[\Gamma]$ of discrete countable groups. We study the cones $\Sigma^{2} \mathbb{C}[\Gamma]$ and $\Sigma^{2} \omega(\Gamma)$ in more detail, where $\omega(\Gamma) \subset \mathbb{C}[\Gamma]$ denotes the augmentation ideal; see Section 4 . The situation for $\omega(\Gamma)$ is much more complicated, as the study is closely related to questions about first cohomology with unitary coefficients. We prove that $\Sigma^{2} \omega(\Gamma)$ has an interior point if and only if $H_{1}(\Gamma, \mathbb{C})=0$. The cone $\Sigma^{2} \omega(\Gamma)$ has an interior point in the $\ell^{1}$-metric if $\Gamma$ has Kazhdan's property (T), and the converse holds if $H_{2}(\Gamma, \mathbb{C})=0$ (see Section 5). In Section 6, we analyze the situation for free groups more closely and reprove theorems of Schmüdgen and Helton.

Along the way we prove some new and powerful separation theorems in Sections 2 and 3. The Hahn-Banach separation theorems for convex sets only apply if additional conditions on the involved sets are imposed; sets have to be closed or have to have nonempty interior, etc. We can remove all additional assumptions at the expense of enlarging the target $\mathbb{R}$ to some real closed extension of the real numbers; see Theorem 2.1.

\section{A real closed separation theorem for convex sets}

Throughout, we will work with various real closed fields $\mathbf{R}$ and always assume that $\mathbb{R} \subset \mathbf{R}$. The following is a first general separation theorem for convex cones.

Theorem 2.1. Let $V$ be an $\mathbb{R}$-vector space, $C \subset V$ a convex cone and $x \notin C$. Then there exist a real closed field $\mathbf{R}$ containing $\mathbb{R}$ and an $\mathbb{R}$-linear functional $\varphi: V \rightarrow R$ 
such that

$$
\varphi(x)<0 \text { and } \varphi(y) \geq 0 \text { for all } y \in C .
$$

We can even ensure $\varphi(y)>0$ for all $y \in C \backslash(C \cap-C)$. Also, $\mathbf{R}$ depends only on $V$, not on $x$ or $C$.

Proof. Let us first assume that $V$ is finite-dimensional. We construct a complete flag of subspaces $V=H_{1} \supset H_{2} \supset \cdots \supset H_{n}=C \cap-C$, starting with $V=H_{1}$, in the following way. By the standard separation theorem for convex sets (see for example Theorem 2.9 in [Barvinok 2002]), we choose a nontrivial $\mathbb{R}$-linear functional $\varphi_{i}: H_{i} \rightarrow \mathbb{R}$ such that $\varphi_{i}(y) \geq 0$ for all $y \in C \cap H_{i}$ and $\varphi_{i}(x) \leq 0$ (if $\left.x \in H_{i}\right)$. We then define $H_{i+1}:=H_{i} \cap\left\{\varphi_{i}=0\right\}$ and iterate the process. We finally extend each $\varphi_{i}$ in any way to $V$. Now let $\mathbf{R}$ be a proper real closed extension field of $\mathbb{R}$. Choose positive elements

$$
1=\epsilon_{1}>\epsilon_{2}>\cdots>\epsilon_{n-1}>0
$$

from $\mathbf{R}$ such that $k \cdot \epsilon_{i}<\epsilon_{i-1}$ for all $k \in \mathbb{R}$. For example, $\epsilon_{2}$ can be any infinitesimal element with respect to $\mathbb{R}$, which exists since $\mathbf{R}$ is a proper extension of $\mathbb{R}$; the following $\epsilon_{i}$ can be taken as powers of $\epsilon_{2}$.

Then define

$$
\varphi:=\epsilon_{1} \varphi_{1}+\cdots+\epsilon_{n-1} \varphi_{n-1} .
$$

One checks that $\varphi$ has the desired properties. This proves the claim in the case of finite dimension.

In the general case, consider the set $\mathscr{Y}$ of all finite-dimensional subspaces $H$ of $V$. For each $H \in \mathscr{Y}$, choose $\varphi_{H}: H \rightarrow \mathbf{R}$, separating $x$ from $C \cap H$ as desired (if $x \in H)$. Extend $\varphi_{H}$ in any way to $V$. Now let $\omega$ be an ultrafilter on $\mathscr{S}$, containing the sets $\{H \in \mathscr{Y} \mid y \in H\}$ for all $y \in V$. Consider the linear functional $\varphi: V \rightarrow \mathbf{R}^{\omega}$, $\varphi(v)=\left(\varphi_{H}(v)\right)_{H \in \mathscr{S}}$. Here $\mathbf{R}^{\omega}$ denotes the ultrapower of $\mathbf{R}$ with respect to $\omega$. One checks that $\varphi$ separates $x$ from $C$ as desired, by the theorem of $Ł o s$ (see for example Theorem 2.2.9 in [Prestel and Delzell 2001]).

Remark 2.2. In the usual way, one can now also deduce that any two convex disjoint sets in a vector space can be separated as above with a real-closed valued affine functional.

It turns out that we can also extend functionals quite often if we allow for an extension of the real closed field.

Theorem 2.3. Let $V$ be an $\mathbb{R}$-vector space, $C \subseteq V$ a convex cone, and $H \subseteq V$ a subspace. Assume $(C+H) \cap-(C+H)=H$. Then for any real closed extension field $\mathbf{R}$ of $\mathbb{R}$ and any $\mathbb{R}$-linear functional $\varphi: H \rightarrow \mathbf{R}$ with $\varphi \geq 0$ on $C \cap H$, there is a real closed extension field $\mathbf{R}^{\prime}$ of $\mathbf{R}$ and an $\mathbb{R}$-linear functional $\bar{\varphi}: V \rightarrow \mathbf{R}^{\prime}$ with $\bar{\varphi} \geq 0$ on $C$ and $\bar{\varphi}=\varphi$ on $H$. We can even ensure $\bar{\varphi}(y)>0$ for all $y \in C \backslash H$. 
Proof. We apply Theorem 2.1 to the convex cone $C+H$ in $V$ and obtain a real closed field $\widetilde{\mathbf{R}}$ and an $\mathbb{R}$-linear functional $\psi: V \rightarrow \widetilde{\mathbf{R}}$ with $\psi=0$ on $H$ and $\psi(y)>0$ for $a \in C \backslash H$. By amalgamation of real closed fields, we can assume without loss of generality that $\mathbf{R}=\widetilde{\mathbf{R}}$. Finally, let $\mathbf{R}^{\prime}$ be a real closed extension field of $\mathbf{R}$ that contains an element $\delta>\mathbf{R}$. Extend $\varphi$ to an $\mathbf{R}$-valued functional on $V$ and set

$$
\bar{\varphi}:=\varphi+\delta \cdot \psi .
$$

It is clear that $\bar{\varphi}$ coincides with $\varphi$ on $H$ and also that $\bar{\varphi}(y)>0$ for all $y \in C \backslash H$.

We will improve upon the separation results in the case of certain $*$-algebras in the next section.

\section{Completely positive separation}

Throughout this section, let $A$ be a $\mathbb{C}$-algebra with involution $*$, not necessarily unital. We consider the cone of sums of hermitian squares

$$
\Sigma^{2} A=\left\{\sum_{i=1}^{n} a_{i}^{*} a_{i} \mid n \in \mathbb{N}, a_{i} \in A\right\}
$$

contained in the real vector subspace of hermitian elements

$$
A^{h}=\left\{a \in A \mid a^{*}=a\right\} .
$$

If $b \in A^{h} \backslash \Sigma^{2} A$, we find an $\mathbb{R}$-linear functional $\varphi: A^{h} \rightarrow \mathbf{R}$, into some real closed extension field $\mathbf{R}$ of $\mathbb{R}$, such that $\varphi(b)<0, \varphi\left(a^{*} a\right) \geq 0$ for all $a \in A$, by Theorem 2.1. We can extend $\varphi$ uniquely to a $\mathbb{C}$-linear functional $\varphi: A \rightarrow \mathbf{R}[i]$ fulfilling $\varphi\left(a^{*}\right)=\overline{\varphi(a)}$. We will denote the algebraically closed field $\mathbf{R}[i]$ by $\mathbf{C}$ from now on.

The condition $\varphi\left(a^{*} a\right) \geq 0$ for all $a \in A$ is called positivity of $\varphi$. We would now like positive and real-closed valued functionals to fulfill the Cauchy-Schwarz inequality

$$
\left|\varphi\left(a^{*} b\right)\right|^{2} \leq \varphi\left(a^{*} a\right) \varphi\left(b^{*} b\right)
$$

for all $a, b \in A$. However, this is not true in general, as the next example shows.

Example 3.1. Let $A=\mathbb{C}[t]$ be the univariate polynomial ring; $*$ is coefficientwise conjugation. The cone $\Sigma^{2} A$ equals the cone of nonnegative real polynomials. Consider the functional

$$
\varphi: \mathbb{R}[t] \rightarrow \mathbf{R}, \quad p \mapsto p(0)+\epsilon p^{\prime \prime}(0),
$$

where $\epsilon \in \mathbf{R}$ is positive and infinitesimal with respect to $\mathbb{R}$. One checks that $\varphi$ is positive, but for $a=1+t^{2}$ and $b=1$, we have

$$
\left|\varphi\left(a^{*} b\right)\right|^{2}=1+4 \epsilon+4 \epsilon^{2}>1+4 \epsilon=\varphi\left(a^{*} a\right) \varphi\left(b^{*} b\right) .
$$


Example 3.2. The last example can be modified to even fulfill $\varphi\left(a^{*} a\right)>0$ if $a \neq 0$. Indeed, let $1=\epsilon_{0}>\epsilon_{1}>\epsilon_{2}>\cdots>0$ be a sequence of elements from $\mathbf{R}$, such that $\mathbb{R} \cdot \epsilon_{i}<\epsilon_{i-1}$ for all $i$. Then the linear mapping $p \mapsto \sum_{i=0}^{\infty} \epsilon_{i} \cdot p^{(2 i)}(0)$ is well-defined and strictly positive in the desired sense. If we further assume $\mathbb{R} \cdot \epsilon_{2}<\epsilon_{1}^{2}$, then the same argument as in Example 3.1 shows that the Cauchy-Schwarz inequality is not fulfilled.

Definition 3.3. A $\mathbb{C}$-linear functional $\varphi: A \rightarrow \mathbf{C}$ with $\varphi\left(a^{*}\right)=\overline{\varphi(a)}$ is called completely positive if for all $m \in \mathbb{N}$, the componentwise defined function

$$
\varphi^{(m)}: \mathbf{M}_{m}(A) \rightarrow \mathbf{M}_{m}(\mathbf{C})
$$

maps sums of hermitian squares to positive semidefinite matrices.

Remark 3.4. It is easily seen that a positive $\mathbb{C}$-linear functional $\varphi: A \rightarrow \mathbb{C}$ with $\varphi\left(a^{*}\right)=\overline{\varphi(a)}$ is always completely positive.

Example 3.5. The functionals from Examples 3.1 and 3.2 are positive, but not completely positive. Indeed, with $a=1+t^{2}$ and

$$
M=\left(\begin{array}{ll}
1 & a \\
0 & 0
\end{array}\right),
$$

we find that

$$
\varphi^{(2)}\left(M^{*} M\right)=\left(\begin{array}{cc}
1 & \varphi(a) \\
\varphi\left(a^{*}\right) & \varphi\left(a^{*} a\right)
\end{array}\right)
$$

is not positive semidefinite, since its determinant is negative in $\mathbf{R}$.

Lemma 3.6. $A \mathbb{C}$-linear functional $\varphi: A \rightarrow \mathbf{C}$ with $\varphi\left(a^{*}\right)=\overline{\varphi(a)}$ is completely positive if and only if the $\mathbf{C}$-linear extension

$$
\operatorname{id} \otimes \varphi: \mathbf{C} \otimes_{\mathbb{C}} A \rightarrow \mathbf{C}
$$

is positive.

Proof. The condition that id $\otimes \varphi$ is positive is

$$
\begin{aligned}
0 & \leq(\mathrm{id} \otimes \varphi)\left(\left(\sum_{j=1}^{m} z_{j} \otimes a_{j}\right)^{*}\left(\sum_{j=1}^{m} z_{j} \otimes a_{j}\right)\right) \\
& =(\operatorname{id} \otimes \varphi)\left(\sum_{j, k} \bar{z}_{j} z_{k} \otimes a_{j}^{*} a_{k}\right) \\
& =\sum_{j, k} \bar{z}_{j} z_{k} \cdot \varphi\left(a_{j}^{*} a_{k}\right)
\end{aligned}
$$

for all $m \in \mathbb{N}, z_{j} \in \mathbf{C}, a_{j} \in A$. But this just means that the matrix $\left(a_{j}^{*} a_{k}\right)_{j, k}$ is mapped to a positive semidefinite matrix under $\varphi^{(m)}$. Since every sum of hermitian squares in $\mathrm{M}_{m}(A)$ is a finite sum of such rank-one squares, this proves the claim. 
Corollary 3.7. If $\varphi: A \rightarrow \mathbf{C}$ is completely positive, then it fulfills the CauchySchwarz inequality

$$
\left|\varphi\left(a^{*} b\right)\right|^{2} \leq \varphi\left(a^{*} a\right) \varphi\left(b^{*} b\right)
$$

for all $a, b \in A$.

Proof. Either consider the positive and $\mathbf{C}$-linear extension id $\otimes \varphi$ to $\mathbf{C} \otimes \mathbb{C} A$ and use the standard proof for the inequality, or apply $\varphi^{(2)}$ to the sum of hermitian squares

$$
\left(\begin{array}{ll}
a & b \\
0 & 0
\end{array}\right)^{*}\left(\begin{array}{ll}
a & b \\
0 & 0
\end{array}\right)=\left(\begin{array}{ll}
a^{*} a & a^{*} b \\
b^{*} a & b^{*} b
\end{array}\right)
$$

and use that the obtained matrix is positive semidefinite.

Remark 3.8. We see from the last proof that in fact only the 2-positivity of $\varphi$ is needed for the Cauchy-Schwarz inequality.

Corollary 3.9. Let $A$ be a $\mathbb{C}$-algebra with involution and $\mathbf{R}$ a real closed field that contains $\mathbb{R}$. Let $\varphi: A \rightarrow \mathbf{C}$ be a completely positive $\mathbb{C}$-linear functional that satisfies $\varphi\left(a^{*}\right)=\overline{\varphi(a)}$ for all $a \in A$. The gauge $\|a\|_{\varphi}:=\varphi\left(a^{*} a\right)^{1 / 2}$ satisfies

$$
\|\lambda \cdot a\|_{\varphi}=|\lambda| \cdot\|a\|_{\varphi}
$$

and

$$
\|a+b\|_{\varphi} \leq \cdot\|a\|_{\varphi}+\|b\|_{\varphi} .
$$

Proof. The first assertion is obvious. Let's compute

$$
\begin{aligned}
\|a+b\|_{\varphi}^{2} & =\varphi\left((a+b)^{*}(a+b)\right) \\
& =\varphi\left(a^{*} a\right)+\varphi\left(a^{*} b\right)+\varphi\left(b^{*} a\right)+\varphi\left(b^{*} b\right) \\
& \leq\|a\|_{\varphi}^{2}+\|b\|_{\varphi}^{2}+2\left|\varphi\left(a^{*} b\right)\right| \\
& \leq\|a\|_{\varphi}^{2}+\|b\|_{\varphi}^{2}+2\|a\|_{\varphi}\|b\|_{\varphi} \\
& =\left(\|a\|_{\varphi}+\|b\|_{\varphi}\right)^{2} .
\end{aligned}
$$

This proves the claim.

It turns out that separation from the cone of sums of hermitian squares can often be done with a completely positive functional.

Definition 3.10. Let $A$ be a $\mathbb{C}$-algebra with involution, not necessarily unital. Then $A$ is called real reduced if $\sum_{i} a_{i}^{*} a_{i}=0$ implies $a_{i}=0$ for all $i$ and $a_{i} \in A$.

Theorem 3.11. Let $A$ be $a \mathbb{C}$-algebra with involution that is real reduced. Let $b \in A^{h} \backslash \Sigma^{2} A$. Then there is real closed extension field $\mathbf{R}$ of $\mathbb{R}$ and a completely positive $\mathbb{C}$-linear functional $\varphi: A \rightarrow \mathbf{C}$ with $\varphi\left(a^{*}\right)=\overline{\varphi(a)}$ such that

$$
\varphi(b)<0 \text { and } \varphi\left(a^{*} a\right)>0 \text { for } a \in A \backslash\{0\} .
$$


Proof. For any finite-dimensional subspace $H$ of $A$, denote by

$$
\Sigma^{2} H=\left\{\sum_{i} a_{i}^{*} a_{i} \mid a_{i} \in H\right\}
$$

the set of sums of hermitian squares of elements from $H$. It is well known that $\Sigma^{2} H$ is a closed convex cone in a finite-dimensional subspace of $A^{h}$. This follows from the fact that $A$ is real reduced, using for example the approach from [Powers and Scheiderer 2001, Lemma 2.7]. It also follows that $\Sigma^{2} H$ is salient, that is, it fulfills

$$
\Sigma^{2} H \cap-\Sigma^{2} H=\{0\} .
$$

So for each such $H$, there is an $\mathbb{R}$-linear functional $\varphi_{H}: A^{h} \rightarrow \mathbb{R}$ with

$$
\varphi_{H}(b)<0 \text { and } \varphi_{H}\left(a^{*} a\right)>0 \text { for all } a \in H \backslash\{0\} .
$$

Let $\mathscr{Y}$ be the set of all finite-dimensional subspaces $H$ of $A$, equipped with an ultrafilter $\omega$ containing all sets $\{H \in \mathscr{Y} \mid c \in H\}$ for $c \in A$. Define

$$
\varphi: A^{h} \rightarrow \mathbb{R}^{\omega}, \quad \varphi(a):=\left(\varphi_{H}(a)\right)_{H \in \mathscr{S}} .
$$

Then $\varphi$ does the separation as desired. We consider the $\mathbb{C}$-linear extension of $\varphi$ to $A$, and finally show that it is completely positive. The $\mathbb{C}$-linear extension of $\varphi_{H}$ to $A$ indeed maps a matrix $\left(a_{i}^{*} a_{j}\right)_{i, j} \in \mathrm{M}_{n}(A)$ to a positive semidefinite hermitian matrix, at least if all $a_{i} \in H$, as is easily checked (compare to Remark 3.4). Since we can check positivity of the matrix $\left(\varphi\left(a_{i}^{*} a_{j}\right)\right)_{i, j} \in \mathrm{M}_{n}\left(\mathbb{R}^{\omega}[i]\right)$ componentwisely in $\mathrm{M}_{n}(\mathbb{R}[i])$, by the theorem of $€$ os, this finishes the proof.

Throughout, we will take the freedom to consider $*$-representations of $A$ on vector spaces that carry a sesquilinear $\mathbf{C}$-valued inner product, where $\mathbf{C}=\mathbf{R}[i]$ for some real closed field $\mathbf{R} \supset \mathbb{R}$. We call these representations generalized representations. For every completely positive functional $\varphi: A \rightarrow \mathbf{C}$, we can perform the usual GNS construction to construct such a representation (see the proof of Theorem 6.1 below for more technical details). The usual concepts of self-adjointness and positive semidefiniteness of operators on such a vector space can be defined without any problems. The first consequence is the following Positivstellensatz (compare to the standard Positivstellensatz from the introduction):

Theorem 3.12. Let $A$ be a real reduced $*$-algebra, and $a \in A^{h}$. Then a is positive semidefinite in every generalized representation if and only if $a \in \Sigma^{2} A$.

Proof. If $a \notin \Sigma^{2} A$, then there exists a completely positive map $\varphi: A \rightarrow \mathbf{C}$ such that $\varphi(a)<0$. Clearly, $a$ will not be positive semidefinite in the generalized GNS representation associated with $\varphi$. 
Examples for real reduced $*$-algebras are group algebras $\mathbb{C}[\Gamma]$. In Section 6, we will see that for particular groups, the study of generalized representations of $\mathbb{C}[\Gamma]$ can be reduced to the study of usual (finite-dimensional) unitary representations, using Tarski's transfer principle.

\section{Sums of squares in the group algebra}

Let $\Gamma$ be a group and let $\mathbb{C}[\Gamma]$ denote the complex group algebra. A typical element in $\mathbb{C}[\Gamma]$ is denoted by $a=\sum_{g} a_{g} g$, where only finitely many of the $a_{g} \in \mathbb{C}$ are not zero. In $\mathbb{C}[\Gamma]$ we identify $\mathbb{C}$ with $\mathbb{C} \cdot e$, where $e$ denotes the neutral element of $\Gamma$. The group algebra comes equipped with an involution $\left(\sum_{g} a_{g} g\right)^{*}:=\sum_{g} \bar{a}_{g} g^{-1}$ and a trace $\tau: \mathbb{C}[\Gamma] \rightarrow \mathbb{C}$ that is given by the formula $\tau\left(\sum_{g} a_{g} g\right)=a_{e}$. The faithfulness of the trace shows that $\mathbb{C}[\Gamma]$ is real reduced. Let $\Sigma^{2} \mathbb{C}[\Gamma]$ denote the set of sums of hermitian squares in $\mathbb{C}[\Gamma]$. The following appears for example as Example 3 in [Cimprič 2009]:

Remark 4.1. $\|a\|_{1}^{2}-a^{*} a \in \Sigma^{2} \mathbb{C}[\Gamma]$ for all $a \in \mathbb{C}[\Gamma]$, where $\|a\|_{1}=\sum_{g}\left|a_{g}\right|$.

Remark 4.2. From the identity

$$
2\|a\|_{1} \cdot\left(\|a\|_{1}-a\right)=\left(\|a\|_{1}-a\right)^{*}\left(\|a\|_{1}-a\right)+\left(\|a\|_{1}^{2}-a^{*} a\right)
$$

for $a \in \mathbb{C}[\Gamma]^{h}$, we see that 1 is an algebraic interior point of the cone $\Sigma^{2} \mathbb{C}[\Gamma]$ in the real vector space $\mathbb{C}[\Gamma]^{h}$. That means $1+\epsilon a \in \Sigma^{2} \mathbb{C}[\Gamma]$ for all $a \in \mathbb{C}[\Gamma]^{h}$ and sufficiently small $\epsilon>0$. In fact, the $\epsilon$ does only depend on $\|a\|_{1}$ here.

Remark 4.3. As explained in the introduction, for any element $a \in \mathbb{C}[\Gamma]^{h}$ that is positive semidefinite in each (usual) $*$-representation of $\mathbb{C}[\Gamma]$, one thus has $a+\epsilon \in \Sigma^{2} \mathbb{C}[\Gamma]$, for all $\epsilon>0$.

Remark 4.4. Since $\mathbb{C}[\Gamma]$ is real reduced and unital, the result of Theorem 3.12 holds here as well. So if $a$ is positive semidefinite in each generalized representation, then $a \in \Sigma^{2} \mathbb{C}[\Gamma]$.

We now consider the augmentation homomorphism $\varepsilon: \mathbb{C}[\Gamma] \rightarrow \mathbb{C}$, which is defined by $\varepsilon\left(\sum_{g} a_{g} g\right)=\sum_{g} a_{g}$. The augmentation ideal is

$$
\omega(\Gamma):=\operatorname{ker}(\varepsilon)=\left\{a \in \mathbb{C}[\Gamma] \mid \sum_{g} a_{g}=0\right\} .
$$

We set $c(g):=g-1$ and note that $\{c(g) \mid g \in \Gamma \backslash\{e\}\}$ is a basis of $\omega(\Gamma)$. The multiplication satisfies

$$
c(g) c(h)=c(g h)-c(g)-c(h) .
$$


We denote by $\omega^{2}(\Gamma)$ the square of $\omega(\Gamma)$, that is, $\omega^{2}(\Gamma)=\operatorname{span}_{\mathbb{C}}\{a b \mid a, b \in \omega(\Gamma)\}$. Inside $\omega(\Gamma)$, we study the cone of sums of hermitian squares

$$
\Sigma^{2} \omega(\Gamma):=\left\{\sum a_{i}^{*} a_{i} \mid a_{i} \in \omega(\Gamma)\right\} .
$$

We are interested in interior points of this cone. Note that $\Sigma^{2} \omega(\Gamma) \subset \omega^{2}(\Gamma)$ and $\omega(\Gamma) / \omega^{2}(\Gamma)=\mathbb{C} \otimes_{\mathbb{Z}} \Gamma_{a b}$, where $\Gamma_{a b}=\Gamma /[\Gamma, \Gamma]$ and $[\Gamma, \Gamma]$ denotes the subgroup of $\Gamma$ generated by commutators. Hence, if $\Gamma$ has nontorsion abelianization, then $\Sigma^{2} \omega(\Gamma)$ is contained in a proper subspace of $\omega(\Gamma)$. However, we will show below that $\Sigma^{2} \omega(\Gamma)$ always has an interior point in $\omega^{2}(\Gamma)^{h}$.

Lemma 4.5. For any group $\Gamma$, we have $\Sigma^{2} \omega(\Gamma)=\Sigma^{2} \mathbb{C}[\Gamma] \cap \omega(\Gamma)$.

Proof. The inclusion $\Sigma^{2} \omega(\Gamma) \subset \Sigma^{2} \mathbb{C}[\Gamma] \cap \omega(\Gamma)$ is obvious. If $\sum_{i} a_{i}^{*} a_{i} \in \omega(\Gamma)$ with $a_{i} \in \mathbb{C}[\Gamma]$, then $\sum_{i}\left|\varepsilon\left(a_{i}\right)\right|^{2}=0$, and hence $\varepsilon\left(a_{i}\right)=0$ for all $i$. This proves the converse inclusion.

Remark 4.6. It turns out that we can always extend positive functionals $\varphi$ on $\omega(\Gamma)$ to positive functionals $\bar{\varphi}$ on $\mathbb{C}[\Gamma]$, at least if we allow for an extension of the real closed field. Indeed, observe that

$$
\left(\Sigma^{2} \mathbb{C}[\Gamma]+\omega(\Gamma)^{h}\right) \cap-\left(\Sigma^{2} \mathbb{C}[\Gamma]+\omega(\Gamma)^{h}\right)=\omega(\Gamma)^{h},
$$

which follows immediately from an application of the augmentation homomorphism $\varepsilon$. We can thus apply Theorem 2.3 .

Lemma 4.7. Let $\mathbf{R}$ be a real closed extension field of $\mathbb{R}$, and $\varphi: \omega(\Gamma) \rightarrow \mathbf{C} a$ completely positive $\mathbb{C}$-linear functional with $\varphi\left(a^{*}\right)=\overline{\varphi(a)}$ for all $a \in \omega(\Gamma)$. Then for all $s, h \in \Gamma$,

$$
\begin{aligned}
\left|\varphi\left(c(s)^{*} c(h)\right)\right| & \leq \frac{1}{\sqrt{2}} \cdot\left(\varphi\left(c(s)^{*} c(s)\right)+\varphi\left(c(h)^{*} c(h)\right)\right), \\
\varphi\left(c(s h)^{*} c(s h)\right) & \leq 2 \cdot\left(\varphi\left(c(s)^{*} c(s)\right)+\varphi\left(c(h)^{*} c(h)\right)\right) .
\end{aligned}
$$

Proof. The first inequality is an application of the Cauchy-Schwarz inequality (which is fulfilled by completely positive functionals) and the inequality $\lambda \mu \leq(\lambda+$ $\mu)^{2} / 2$. For the second inequality, first apply the triangle identity from Corollary 3.9 to the equation

$$
c(s h)=c(s)+(c(s) c(h)+c(h)),
$$

together with the well-known inequality $(a+b)^{2} \leq 2\left(a^{2}+b^{2}\right)$. Then use the easily verified identity $\|c(s) c(h)+c(h)\|^{2}=\|c(h)\|^{2}$.

Since $1 \notin \omega(\Gamma)$, we need to find a different candidate for an interior point of $\Sigma^{2} \omega(\Gamma)$. 
Definition 4.8. Let $S \subset \Gamma$ be a finite symmetric set, that is, $S^{-1}=S$. We define the Laplace operator on $S$ to be

$$
\Delta(S):=|S|-\sum_{s \in S} s .
$$

Remark 4.9. Note that for every finite symmetric set $S \subset \Gamma$,

$$
\Delta(S)=\frac{1}{2} \cdot \sum_{s \in S} c(s)^{*} c(s) \in \Sigma^{2} \omega(\Gamma) .
$$

Proposition 4.10. Let $\Gamma$ be a group generated by a finite symmetric set $S$. Then for any $b \in \omega^{2}(\Gamma)$, there exists a constant $C(b) \in \mathbb{R}$ such that for any real closed extension field $\mathbf{R}$ of $\mathbb{R}$ and any completely positive $\mathbb{C}$-linear functional $\varphi: \omega(\Gamma) \rightarrow \mathbf{C}$ with $\varphi\left(a^{*}\right)=\overline{\varphi(a)}$ for all $a \in \omega(\Gamma)$, one has

$$
|\varphi(b)| \leq C(b) \cdot \varphi(\Delta(S)) .
$$

Proof. Every element $b \in \omega^{2}(\Gamma)$ is a finite linear combination of $c(g)^{*} c(h)$ for $g, h \in \Gamma \backslash\{e\}$. This implies that $|\varphi(b)|$ is bounded by a constant times

$$
\max \left\{\varphi\left(c(s)^{*} c(s)\right) \mid s \in S\right\},
$$

using Lemma 4.7 several times. However,

$$
\max \left\{\varphi\left(c(s)^{*} c(s)\right) \mid s \in S\right\} \leq 2 \cdot \varphi(\Delta(S))
$$

follows from Remark 4.9. This proves the claim.

Theorem 4.11. Let $\Gamma$ be a group with finite generating symmetric set $S$. Then for every $b \in \omega^{2}(\Gamma)^{h}$, there is a constant $C(b) \in \mathbb{R}$ such that

$$
C(b) \cdot \Delta(S) \pm b \in \Sigma^{2} \omega(\Gamma) .
$$

In particular, $\Delta(S)$ is an inner point of the cone $\Sigma^{2} \omega(\Gamma)$ in $\omega^{2}(\Gamma)^{h}$. If

$$
H_{1}(\Gamma, \mathbb{C})=0,
$$

it is an inner point in $\omega(\Gamma)^{h}$.

Proof. In view of Proposition 4.10, we find that

$$
C(b) \cdot \Delta(S) \pm b
$$

is nonnegative under each completely positive real-closed valued $\mathbb{C}$-linear functional $\varphi$ on $\omega(\Gamma)$. In view of Theorem 3.12, this means that $C(b) \cdot \Delta(S) \pm b$ is a sum of hermitian squares in $\omega(\Gamma)$. Note that we use that $\omega(\Gamma)$ is real reduced. Finally note that $H_{1}(\Gamma, \mathbb{C})=0$ just means that $\omega^{2}(\Gamma)=\omega(\Gamma)$. 


\section{Groups with Kazhdan's property (T)}

We want to show that the constant $C(b)$ from Theorem 4.11 can be chosen as a fixed multiple of $\|b\|_{1}$, in case the group $\Gamma$ has Kazhdan's property.

Definition 5.1. Let $\Gamma$ be a group and $\pi: \Gamma \rightarrow U(H)$ a unitary representation on a Hilbert space $H$.

(1) A 1-cocycle with respect to the unitary representation $\pi$ is a map $\delta: \Gamma \rightarrow H$ such that for all $g, h \in \Gamma$, we have $\delta(g h)=\pi(g) \delta(h)+\delta(g)$.

(2) A 1-cocycle $\delta: \Gamma \rightarrow H$ is called inner if $\delta(g)=\pi(g) \xi-\xi$ for some vector $\xi \in H$.

Definition 5.2. A group has Kazhdan's property $(\mathrm{T})$ if every 1-cocycle with respect to every unitary representation is inner.

We will use several results on Kazhdan groups, which can be found, for example, in [Bekka et al. 2008]. It is well known that groups with Kazhdan's property (T) admit a finite generating set $S$, and that

$$
\omega^{2}(\Gamma)=\omega(\Gamma)
$$

holds. It is also known that for a fixed finite symmetric and generating set $S$ in a Kazhdan group $\Gamma$, there is some $\epsilon>0$ such that for any unitary representation $\pi: \Gamma \rightarrow U(H)$ without nonzero fixed vectors, one has

$$
\langle\Delta(S) \xi, \xi\rangle \geq \epsilon \cdot\|\xi\|^{2} \text { for all } \xi \in H .
$$

Such $\epsilon$ is called a Kazhdan constant for $S$.

Let's revisit the standard GNS representation in the context of $\omega(\Gamma)$. Let

$$
\varphi: \omega(\Gamma) \rightarrow \mathbb{C}
$$

be a positive linear functional with $\varphi\left(a^{*}\right)=\overline{\varphi(a)}$. We associate to $\varphi$ a Hilbert space as follows. We define on $\omega(\Gamma)$ a positive semidefinite sesquilinear form

$$
\langle a, b\rangle_{\varphi}:=\varphi\left(b^{*} a\right)
$$

and set $\|a\|_{\varphi}:=\langle a, a\rangle_{\varphi}^{1 / 2}$. Let $N(\varphi):=\left\{a \in \omega(\Gamma) \mid\|a\|_{\varphi}=0\right\}$ and define $L^{2}(\omega(\Gamma), \varphi)$ to be the metric completion of $\omega(\Gamma) / N(\varphi)$ with respect to $\|\cdot\|_{\varphi}$. We denote the image of $c(g)$ in $L^{2}(\omega(\Gamma), \varphi)$ by $\delta(g)$ and denote by $\delta(\Gamma)$ their complex linear span, which is dense by definition of $\left.L^{2}(\omega(\Gamma), \varphi)\right)$. It is standard that the leftmultiplication of $\omega(\Gamma)$ on itself extends to a homomorphism $\pi^{\varphi}: \omega(\Gamma) \rightarrow D(\delta(\Gamma))$, where $D(\delta(\Gamma))$ denotes the algebra of densely defined linear operators mapping $\delta(\Gamma)$ into itself. Indeed, if $a \in N(\varphi)$ and $b \in \omega(\Gamma)$, then $b a \in N(\varphi)$ since

$$
\varphi\left((b a)^{*} b a\right)=\varphi\left(a^{*} b^{*} b a\right) \leq\|b\|_{1}^{2} \cdot \varphi\left(a^{*} a\right),
$$


by Remark 4.1. Note that

$$
\pi^{\varphi}(c(g)) \delta(h)=\delta(g h)-\delta(g)-\delta(h) .
$$

Now we define a unitary representation $\pi_{\varphi}$ of $\Gamma$ on $L^{2}(\omega(\Gamma), \varphi)$ by the rule

$$
\pi_{\varphi}(g):=\pi^{\varphi}(c(g))+1_{L^{2}(\omega(\Gamma), \varphi)} .
$$

Lemma 5.3. If $\omega^{2}(\Gamma)=\omega(\Gamma)$, then the representation $\pi_{\varphi}$ has no fixed vectors.

Proof. Assume $\eta \in L^{2}(\omega(\Gamma), \varphi)$ is a fixed vector. By definition of $\pi_{\varphi}$, this means $\pi^{\varphi}(c(g)) \eta=0$ for all $g \in \Gamma$. Hence,

$$
0=\left\langle\pi^{\varphi}\left(c\left(g^{-1}\right)\right) \eta, \delta(h)\right\rangle_{\varphi}=\langle\eta, \delta(g h)-\delta(g)-\delta(h)\rangle_{\varphi} .
$$

Since $c(g) c(h)=c(g h)-c(g)-c(h)$, the vectors $\delta(g h)-\delta(g)-\delta(h)$ span the image of $\omega^{2}(\Gamma)$ in $\delta(\Gamma)$, and hence $\delta(\Gamma)$, since $\omega^{2}(\Gamma)=\omega(\Gamma)$.

Note that the map $g \mapsto \delta(g)$ satisfies

$$
\delta(g h)=\pi_{\varphi}(g) \delta(h)+\delta(g),
$$

and hence defines a 1-cocycle with respect to the representation $\pi_{\varphi}$. If $\Gamma$ is a Kazhdan group, then there exists $\Omega \in L^{2}(\omega(\Gamma), \varphi)$ such that

$$
\delta(g)=\pi_{\varphi}(g) \Omega-\Omega .
$$

Proposition 5.4. Let $\Gamma$ be a Kazhdan group with finite symmetric generating set $S$ and Kazhdan constant $\epsilon>0$. Then for every nonzero $b \in \omega(\Gamma)^{h}$, every real closed extension field $\mathbf{R}$ of $\mathbb{R}$, and every positive nontrivial $\mathbb{C}$-linear functional $\varphi: \omega(\Gamma) \rightarrow \mathbf{C}$ with $\varphi\left(a^{*}\right)=\overline{\varphi(a)}$, one has

$$
\epsilon \cdot \varphi(b)<2\|b\|_{1} \cdot \varphi(\Delta(S)) .
$$

Proof. Let us first assume $\mathbf{R}=\mathbb{R}$ and $\mathbf{C}=\mathbb{C}$. We do the GNS construction as just described, and get some $\Omega \in L^{2}(\omega(\Gamma), \varphi)$ with $\delta(g)=\pi_{\varphi}(g) \Omega-\Omega$, for all $g \in \Gamma$. We set

$$
\bar{\varphi}: \mathbb{C}[\Gamma] \rightarrow \mathbb{C}, \quad \bar{\varphi}(a)=\left\langle\pi_{\varphi}(a) \Omega, \Omega\right\rangle
$$

and compute

$$
\bar{\varphi}\left(c(h)^{*} c(g)\right)=\left\langle\pi_{\varphi}(c(g)) \Omega, \pi_{\varphi}(c(h)) \Omega\right\rangle_{\varphi}=\langle\delta(g), \delta(h)\rangle_{\varphi}=\varphi\left(c(h)^{*} c(g)\right) .
$$

This shows that $\bar{\varphi}$ and $\varphi$ agree on $\omega^{2}(\Gamma)$, and hence on $\omega(\Gamma)$. If we now do the standard GNS construction with respect to $\bar{\varphi}$, we see that there is a natural $\Gamma$ equivariant identification of $L^{2}(\mathbb{C}[\Gamma], \bar{\varphi})$ and $L^{2}(\omega(\Gamma), \varphi)$. Since the representation $\pi_{\varphi}$ has no fixed vectors, we get

$$
\varphi(\Delta(S))=\bar{\varphi}(\Delta(S))=\langle\Delta(S) 1,1\rangle_{\bar{\varphi}} \geq \epsilon \cdot \bar{\varphi}(1) .
$$


Since $\bar{\varphi}$ is positive and nontrivial, it follows from Remark 4.2 that $\bar{\varphi}(1)>0$. So finally, again using Remark 4.2, we find

$$
\epsilon \cdot \varphi(b)=\epsilon \cdot \bar{\varphi}(b) \leq \epsilon \cdot\|b\|_{1} \cdot \bar{\varphi}(1)<2\|b\|_{1} \cdot \varphi(\Delta(S)),
$$

the desired result.

Now let $\mathbf{R}$ be arbitrary, and let $\varphi: \omega(\Gamma) \rightarrow \mathbf{C}$ be positive and nontrivial. From Theorem 4.11 it follows that $\varphi(\Delta(S))>0$. So we can assume without loss of generality that $\varphi(\Delta(S))=1$. Again from Theorem 4.11, we see that $\varphi$ now only takes values in $\mathcal{O}[i]$, where $\mathcal{O}$ is the convex hull of $\mathbb{R}$ in $\mathbf{R}$. It is well known that $\mathcal{O}$ is a valuation ring in $\mathbf{R}$ with maximal ideal $\mathfrak{m}$, and that $0 / \mathfrak{m}=\mathbb{R}$ (see for example [Prestel and Delzell 2001], especially the appendix on valued fields). The residue map $\mathbb{O} \rightarrow \mathbb{O} / \mathfrak{m}$ maps nonnegative elements to nonnegative elements. So if we compose $\varphi$ with the residue map on $\mathbb{O}[i]$, we get a positive linear functional to $\mathbb{C}$. Since we know that the desired strict inequality holds now, it was already valid for $\varphi$.

Theorem 5.5. Let $\Gamma$ be a group with finite generating symmetric set $S$. Consider the statements:

(1) $\Gamma$ has Kazhdan's property $(T)$.

(2) $\Delta(S)$ is an algebraic interior point of $\Sigma^{2} \omega(\Gamma)$ in the $\ell^{1}$-metric of $\omega(\Gamma)^{h}$. More precisely, there exists a constant $\epsilon>0$ such that for every $b \in \omega(\Gamma)^{h}$ with $\|b\|_{1}=1$, we have

$$
\Delta(S)+\epsilon \cdot b \in \Sigma^{2} \omega(\Gamma) .
$$

The following implications hold: (1) implies (2), and (2) implies (1) under the additional assumption $\mathrm{H}_{2}(\Gamma, \mathbb{C})=0$.

Proof. The implication (1) $\Rightarrow(2)$ is a direct consequence of Theorem 3.11 and Proposition 5.4. Let us now prove $(2) \Rightarrow(1)$ under the additional assumption $H_{2}(\Gamma, \mathbb{C})=0$. We first prove two lemmas.

Lemma 5.6. Let $\Gamma$ be a group. There is an exact sequence as follows:

$$
0 \rightarrow H_{2}(\Gamma, \mathbb{C}) \rightarrow \omega(\Gamma) \otimes_{\mathbb{C}[\Gamma]} \omega(\Gamma) \rightarrow \omega(\Gamma) \rightarrow H_{1}(\Gamma, \mathbb{C}) \rightarrow 0 .
$$

Proof. It is well known that

$$
\omega(\Gamma) / \omega^{2}(\Gamma)=\Gamma_{a b} \otimes_{\mathbb{Z}} \mathbb{C}=H_{1}(\Gamma, \mathbb{C}) .
$$

This shows exactness at $\omega(\Gamma)$, and it remains to show exactness at $\omega(\Gamma) \otimes \mathbb{C}[\Gamma] \omega(\Gamma)$. Since $0 \rightarrow \omega(\Gamma) \rightarrow \mathbb{C}[\Gamma] \rightarrow \mathbb{C} \rightarrow 0$ is exact, we have

$$
H_{2}(\Gamma, \mathbb{C})=H_{1}(\Gamma, \omega(\Gamma))=\operatorname{Tor}_{1}^{\mathbb{C}[\Gamma]}(\mathbb{C}, \omega(\Gamma)) .
$$


Again, we get an exact sequence

$\operatorname{Tor}_{1}^{\mathbb{C}[\Gamma]}(\mathbb{C}[\Gamma], \omega(\Gamma)) \rightarrow \operatorname{Tor}_{1}^{\mathbb{C}[\Gamma]}(\mathbb{C}, \omega(\Gamma)) \rightarrow \omega(\Gamma) \otimes_{\mathbb{C}[\Gamma]} \omega(\Gamma) \rightarrow \mathbb{C}[\Gamma] \otimes_{\mathbb{C}[\Gamma]} \omega(\Gamma)$.

This finishes the proof, since $\operatorname{Tor}_{1}^{\mathbb{C}[\Gamma]}(\mathbb{C}[\Gamma], \omega(\Gamma))=0$.

If $\Delta(S)$ is an algebraic interior point of $\Sigma^{2} \omega(\Gamma)$ in $\omega(\Gamma)^{h}$, then $\omega(\Gamma)=\omega^{2}(\Gamma)$, that is, $H_{1}(\Gamma, \mathbb{C})=0$. Hence $H_{2}(\Gamma, \mathbb{C})=0$ ensures that the natural map

$$
\omega(\Gamma) \otimes_{\mathbb{C}[\Gamma]} \omega(\Gamma) \rightarrow \omega(\Gamma)
$$

is an isomorphism. This is what we are going to use.

Lemma 5.7. Let $\pi: \Gamma \rightarrow U(H)$ be a unitary representation and let $\delta: \Gamma \rightarrow H$ be a 1-cocycle with respect to $H$. Then

$$
\varphi\left(c(h)^{*} \otimes c(g)\right):=\langle\delta(g), \delta(h)\rangle
$$

yields a well-defined positive linear functional on $\omega(\Gamma)=\omega(\Gamma) \otimes_{\mathbb{C}[\Gamma]} \omega(\Gamma)$.

Proof. It is clear that $\left(c(h)^{*}, c(g)\right) \mapsto\langle\delta(g), \delta(h)\rangle$ defines a bilinear map on $\omega(\Gamma)$, that is, a linear map $\varphi^{\prime}: \omega(\Gamma) \otimes_{\mathbb{C}} \omega(\Gamma) \rightarrow \mathbb{C}$. We show that this map passes to $\omega(\Gamma) \otimes_{\mathbb{C}[\Gamma]} \omega(\Gamma)$. Let $g, h, k \in \Gamma$; then

$$
\begin{aligned}
\varphi^{\prime}\left(c(h)^{*} k \otimes c(g)\right) & =\varphi^{\prime}\left(c\left(h^{-1}\right) k \otimes c(g)\right) \\
& =\varphi^{\prime}\left(\left(c\left(h^{-1} k\right)-c(k)\right) \otimes c(g)\right) \\
& =\varphi^{\prime}\left(c\left(k^{-1} h\right)^{*} \otimes c(g)\right)-\varphi^{\prime}\left(c\left(k^{-1}\right)^{*} \otimes c(g)\right) \\
& =\left\langle\delta(g), \delta\left(k^{-1} h\right)\right\rangle-\left\langle\delta(g), \delta\left(k^{-1}\right)\right\rangle \\
& =\left\langle\delta(g), \pi\left(k^{-1}\right) \delta(h)\right\rangle \\
& =\langle\pi(k) \delta(g), \delta(h)\rangle \\
& =\langle\delta(k g), \delta(h)\rangle-\langle\delta(k), \delta(h)\rangle \\
& =\varphi^{\prime}\left(c(h)^{*} \otimes c(k g)\right)-\varphi^{\prime}\left(c(h)^{*} \otimes c(k)\right) \\
& =\varphi^{\prime}\left(c\left(h^{*}\right) \otimes k c(g)\right) .
\end{aligned}
$$

We can now understand $\varphi^{\prime}$ as a linear map on $\omega(\Gamma)$ via the above isomorphism to $\omega(\Gamma) \otimes_{\mathbb{C}[\Gamma]} \omega(\Gamma)$. Since $a^{*} a$ corresponds to $a^{*} \otimes a$, one easily checks that $\varphi$ is positive on $\omega(\Gamma)$.

We continue with the proof of Theorem 5.5. Condition (2) in Theorem 5.5 and Lemma 5.7 imply that any 1-cocycle with respect to any unitary representation is bounded. This is well known to imply Kazhdan's property $(\mathrm{T})$ for $\Gamma$.

Remark 5.8. It is not clear whether the condition $H_{2}(\Gamma, \mathbb{C})=0$ is necessary. 
There is an analogue of the implication $(1) \Rightarrow(2)$ in Theorem 5.5 in

$$
\ell^{1} \Gamma:=\left\{\sum_{g \in \Gamma} a_{g} g\left|\sum_{g \in \Gamma}\right| a_{g} \mid<\infty\right\} .
$$

We set $\omega^{1} \Gamma:=\overline{\omega(\Gamma)}^{\|\cdot\|_{1}}$ and define

$$
\Sigma^{2,1} \omega(\Gamma):=\left\{\sum_{i=1}^{\infty} a_{i}^{*} a_{i} \mid a_{i} \in \omega[\Gamma], \sum_{i=1}^{\infty}\left\|a_{i}\right\|_{1}^{2}<\infty\right\}
$$

and

$$
\Sigma^{2,1} \ell^{1}(\Gamma):=\left\{\sum_{i=1}^{\infty} a_{i}^{*} a_{i} \mid a_{i} \in \ell^{1}[\Gamma], \sum_{i=1}^{\infty}\left\|a_{i}\right\|_{1}^{2}<\infty\right\} .
$$

We note that $\|a\|_{1}-a \in \Sigma^{2,1} \ell^{1}(\Gamma)$ for every hermitian element $a \in \ell^{1} \Gamma$. Indeed, $\|a\|_{1}-a=\sum_{g \in G} 2\left|a_{g}\right|-a_{g} g-\bar{a}_{g} g^{-1}=\sum_{g \in G}\left(\left|a_{g}\right|^{1 / 2}-\frac{a_{g}}{\left|a_{g}\right|^{1 / 2}} g\right)^{*}\left(\left|a_{g}\right|^{1 / 2}-\frac{a_{g}}{\left|a_{g}\right|^{1 / 2}} g\right)$.

Hence, for $\varphi: \ell^{1} \Gamma \rightarrow \mathbf{C}, \mathbb{C}$-linear and positive on $\Sigma^{2,1} \ell^{1} \Gamma,|\varphi(a)| \leq\|a\|_{1}$ for all $a \in \ell^{1} \Gamma$. A priori, there is no reason to assume that $\Sigma^{2,1} \ell^{1} \Gamma$ or $\Sigma^{2,1} \omega^{1} \Gamma$ are closed or have nontrivial interior. Nevertheless, our result shows:

Corollary 5.9. Let $\Gamma$ be a Kazhdan group with finite generating symmetric set $S$ and Kazhdan constant $\epsilon$. Then for every $b \in \omega^{1}(\Gamma)^{h}$ with $\|b\|_{1}=1$, we have

$$
\Delta(S)+\varepsilon \cdot b \in \Sigma^{2,1} \omega(\Gamma) .
$$

\section{Group algebras of free groups}

In this section, let $\Gamma=F_{n}$ be the free group on $n$ generators $g_{1}, \ldots, g_{n}$ or $\Gamma=$ $F_{\infty}$. Schmüdgen (private communication, 2011) has proven that an element from the group algebra $\mathbb{C}[\Gamma]$ that is nonnegative under each finite-dimensional *-representation is a sum of squares. We demonstrate how his result can be reproved with our real closed separation approach. The main idea of our proof is the same as in Schmüdgen's work. However, instead of a partial GNS construction, we use a full GNS construction, but over a general real closed field. We then reduce to the standard real numbers by Tarski's transfer principle.

Theorem 6.1 (Schmüdgen). Let $\Gamma=F_{n}$ be the free group on $n$ generators. If $b \in \mathbb{C}[\Gamma]^{h}$ is mapped to a positive semidefinite matrix under each finite-dimensional $*$-representation of $\mathbb{C}[\Gamma]$, then $b \in \Sigma^{2} \mathbb{C}[\Gamma]$.

Proof. Assume that $b \notin \Sigma^{2} \mathbb{C}[\Gamma]$. By Theorem 3.11, there is a real closed extension field $\mathbf{R}$ of $\mathbb{R}$ and a completely positive $\mathbb{C}$-linear functional $\varphi: \mathbb{C}[\Gamma] \rightarrow \mathbf{C}$ with $\varphi\left(a^{*}\right)=\overline{\varphi(a)}$, such that $\varphi(b)<0$. By Lemma 3.6, the canonical $\mathbf{C}$-linear extension 
of $\varphi$ to $A=\mathbf{C} \otimes \mathbb{C} \mathbb{C}[\Gamma]$ is still positive, and we denote it again by $\varphi$. We apply the usual GNS construction to $A$. We note that

$$
N=\left\{a \in A \mid \varphi\left(a^{*} a\right)=0\right\}
$$

is a $*$-subspace of the $\mathbf{C}$-vector space $A$, which follows from the Cauchy-Schwarz inequality, as shown in Corollary 3.9. We denote the quotient space $A / N$ by $H$, and note that

$$
\langle a+N, c+N\rangle_{\varphi}:=\varphi\left(c^{*} a\right)
$$

is a well-defined and positive definite $\mathbf{C}$-valued sesquilinear form on $H$. We also note that left-multiplication from $\mathbb{C}[\Gamma]$ on $A$ is well-defined on $H$, as explained in Section 5 . So we have a $\mathbb{C}$-linear $*$-representation

$$
\pi: \mathbb{C}[\Gamma] \rightarrow \mathscr{L}(H)
$$

with $\langle\pi(b) \xi, \xi\rangle_{\varphi}<0$, where $\xi=1+N$.

Now let $H^{\prime}$ be a finite-dimensional $*$-subspace of $H$, containing the residue classes of all words in the generators $g_{i}$ of length at most $d$, where $d$ is the maximal word length in $b$. We can choose an orthonormal basis $v_{1}, \ldots v_{m}$ of $H^{\prime}$, using the usual Gram-Schmidt procedure over $\mathbf{C}$. So there is an orthogonal projection map $p: H \rightarrow H^{\prime}$, defined as

$$
p: h \mapsto \sum_{i=1}^{m}\left\langle h, v_{i}\right\rangle v_{i}
$$

Define

$$
M_{i}:=p \circ \pi\left(g_{i}\right) \in \mathscr{L}\left(H^{\prime}\right) .
$$

It is easy to see that all $M_{i}$ are contractions; thus the linear operators $\sqrt{1-M_{i}^{*} M_{i}}$ and $\sqrt{1-M_{i} M_{i}^{*}}$ exist on $H^{\prime}$. Using Choi's matrix trick [1980, Theorem 7], we define

$$
U_{i}:=\left(\begin{array}{cc}
M_{i} & \sqrt{I-M_{i} M_{i}^{*}} \\
\sqrt{I-M_{i}^{*} M_{i}} & -M_{i}^{*}
\end{array}\right) \in \mathscr{L}\left(H^{\prime} \oplus H^{\prime}\right) .
$$

The $U_{i}$ are checked to be unitary operators, and thus yield a $\mathbb{C}$-linear $*$-representation $\tilde{\pi}$ of $\mathbb{C}[\Gamma]$ on $H^{\prime} \oplus H^{\prime}$. Since the residue classes of all words occurring in $b$ belong to $H^{\prime}$, and by the definition of the $U_{i}$, we find

$$
\left\langle\tilde{\pi}(b) \xi^{\prime}, \xi^{\prime}\right\rangle_{H^{\prime} \oplus H^{\prime}}=\langle\pi(b) \xi, \xi\rangle_{\varphi}<0,
$$

where $\xi^{\prime}=(\xi, 0)$. Now finally, since $H^{\prime} \oplus H^{\prime}$ is finite-dimensional, the existence of such a representation over $\mathbf{C}$ implies the existence over $\mathbb{C}$, by Tarski's transfer principle. This finishes the proof.

Remark 6.2. The proof becomes even simpler when considering the $*$-algebra of polynomials in noncommuting variables $\mathbb{C}\left\langle y_{1}, \ldots, y_{n}, z_{1}, \ldots, z_{n}\right\rangle$ with $y_{i}^{*}=z_{i}$, or $\mathbb{C}\left\langle z_{1}, \ldots, z_{n}\right\rangle$ with $z_{i}^{*}=z_{i}$, instead of the group algebra of a free group. The reason 
is that one is not forced to make the matrices $M_{i}$ unitary (only hermitian in the second case). So Theorem 6.1 also holds for these polynomial algebras. This was first proven by Helton [2002].

It is an interesting problem to study the class of groups $\Gamma$ for which positivity of $a \in \mathbb{C}[\Gamma]$ in every finite-dimensional unitary representation implies that $a \in$ $\Sigma^{2} \mathbb{C}[\Gamma]$. It is clear that in order for an analogous argument to work, $\Gamma$ has to be residually finite-dimensional in a very strong sense. Residual finite-dimensionality of a group means that every unitary representation on a Hilbert space can be approximated in the Fell topology by finite-dimensional representations; see [Brown and Ozawa 2008] for details. If - more generally - every generalized unitary representation of $\Gamma$ on a Hilbert space can be approximated on finitely many vectors by generalized finite-dimensional unitary representations, then everything works. With additional work, this can be carried out for virtually free groups (Schmüdgen, private communication).

Deep results of Scheiderer [2006] imply that the conclusion holds for $\mathbb{Z}^{2}$. By a classical result [Rudin 1963], however, the group $\mathbb{Z}^{3}$ does not satisfy the desired conclusion, and the same holds for every group containing $\mathbb{Z}^{3}$. This is also implied by seminal work of Scheiderer [2000, Theorem 6.2], who showed that the existence of positive elements that are not sums of squares under general assumptions in dimension $\geq 3$.

This shows that the theory of generalized unitary representations is fundamentally different and new pathologies occur.

An intriguing and possibly manageable case is that of surface groups. Lubotzky and Shalom [2004] showed that surface groups are residually finite-dimensional. It is quite possible that their methods extend and lead to a resolution of the case of surface groups.

Conjecture 6.3. Let $\Gamma$ be a surface group. Every element $a \in \mathbb{C}[\Gamma]^{h}$ that is positive semidefinite in every finite-dimensional unitary representation lies in $\Sigma^{2} \mathbb{C}[\Gamma]$.

Similar questions can be studied if one allows the unitary representations to be infinite-dimensional. Again, the only known obstruction is $\mathbb{Z}^{3} \subset \Gamma$.

\section{References}

[Barvinok 2002] A. Barvinok, A course in convexity, Graduate Studies in Mathematics 54, American Mathematical Society, Providence, RI, 2002. MR 2003j:52001 Zbl 1014.52001

[Bekka et al. 2008] B. Bekka, P. de la Harpe, and A. Valette, Kazhdan's property (T), New Mathematical Monographs 11, Cambridge University Press, Cambridge, 2008. MR 2009i:22001 Zbl 1146. 22009

[Brown and Ozawa 2008] N. P. Brown and N. Ozawa, $C^{*}$-algebras and finite-dimensional approximations, Graduate Studies in Mathematics 88, American Mathematical Society, Providence, RI, 2008. MR 2009h:46101 Zbl 1160.46001 
[Choi 1980] M. D. Choi, "The full $C^{*}$-algebra of the free group on two generators", Pacific J. Math. 87:1 (1980), 41-48. MR 82b:46069 Zbl 0463.46047

[Cimprič 2009] J. Cimprič, "A representation theorem for Archimedean quadratic modules on *rings”, Canad. Math. Bull. 52:1 (2009), 39-52. MR 2010c:46154 Zbl 1173.16024

[Cimprič et al. 2011] J. Cimprič, M. Marshall, and T. Netzer, "Closures of quadratic modules", Israel J. Math. 183 (2011), 445-474. MR 2012e:13047 Zbl 1235.13021

[Helton 2002] J. W. Helton, “Positive' noncommutative polynomials are sums of squares", Ann. of Math. (2) 156:2 (2002), 675-694. MR 2003k:12002 Zbl 1033.12001

[Lubotzky and Shalom 2004] A. Lubotzky and Y. Shalom, "Finite representations in the unitary dual and Ramanujan groups", pp. 173-189 in Discrete geometric analysis (Sendai, 2002), edited by M. Kotani et al., Contemp. Math. 347, Amer. Math. Soc., Providence, RI, 2004. MR 2005e:22011 Zbl 1080.22006

[Powers and Scheiderer 2001] V. Powers and C. Scheiderer, "The moment problem for non-compact semialgebraic sets", Adv. Geom. 1:1 (2001), 71-88. MR 2002c:14086 Zbl 0984.44012

[Prestel and Delzell 2001] A. Prestel and C. N. Delzell, Positive polynomials: from Hilbert's 17th problem to real algebra, Springer, Berlin, 2001. MR 2002k:13044 Zbl 0987.13016

[Rudin 1963] W. Rudin, "The extension problem for positive-definite functions", Illinois J. Math. 7 (1963), 532-539. MR 27 \#1779 Zbl 0114.31003

[Scheiderer 2000] C. Scheiderer, "Sums of squares of regular functions on real algebraic varieties", Trans. Amer. Math. Soc. 352:3 (2000), 1039-1069. MR 2000j:14090 Zbl 0941.14024

[Scheiderer 2006] C. Scheiderer, "Sums of squares on real algebraic surfaces", Manuscripta Math. 119:4 (2006), 395-410. MR 2006m:14079 Zbl 1120.14047

[Schmüdgen 1991] K. Schmüdgen, "The $K$-moment problem for compact semi-algebraic sets", Math. Ann. 289:2 (1991), 203-206. MR 92b:44011 Zbl 0744.44008

[Schmüdgen 2009] K. Schmüdgen, "Noncommutative real algebraic geometry: some basic concepts and first ideas", pp. 325-350 in Emerging applications of algebraic geometry, edited by M. Putinar and S. Sullivant, IMA Vol. Math. Appl. 149, Springer, New York, 2009. MR 2010m:13036 Zbl 1158.13313

Received January 24, 2012. Revised August 8, 2012.

TIM NETZER

MATHEMATISCHES INSTITUT

UNIVERSITY OF LEIPZIG

PF 100920

D-04009 LEIPZIG

GERMANY

netzer@math.uni-leipzig.de

\author{
ANDREAS THOM \\ MATHEMATISCHES INSTITUT \\ UNIVERSITY OF LEIPZIG \\ PF 100920 \\ D-04009 LEIPZIG \\ GERMANY \\ thom@math.uni-leipzig.de
}




\title{
PACIFIC JOURNAL OF MATHEMATICS
}

\author{
msp.org/pjm
}

Founded in 1951 by E. F. Beckenbach (1906-1982) and F. Wolf (1904-1989)

\section{EDITORS}

V. S. Varadarajan (Managing Editor)

Department of Mathematics

University of California

Los Angeles, CA 90095-1555

pacific@math.ucla.edu

Paul Balmer

Department of Mathematics

University of California

Los Angeles, CA 90095-1555

balmer@math.ucla.edu

Daryl Cooper

Department of Mathematics

University of California

Santa Barbara, CA 93106-3080 cooper@math.ucsb.edu

Jiang-Hua $\mathrm{Lu}$

Department of Mathematics

The University of Hong Kong

Pokfulam Rd., Hong Kong jhlu@maths.hku.hk
Don Blasius

Department of Mathematics University of California

Los Angeles, CA 90095-1555

blasius@math.ucla.edu

Robert Finn

Department of Mathematics Stanford University

Stanford, CA 94305-2125

finn@math.stanford.edu

Sorin Popa

Department of Mathematics

University of California

Los Angeles, CA 90095-1555

popa@math.ucla.edu

Paul Yang

Department of Mathematics

Princeton University

Princeton NJ 08544-1000

yang@math.princeton.edu

\section{PRODUCTION}

Silvio Levy, Scientific Editor, production@msp.org

\section{SUPPORTING INSTITUTIONS}

ACADEMIA SINICA, TAIPEI

CALIFORNIA INST. OF TECHNOLOGY

INST. DE MATEMÁTICA PURA E APLICADA

KEIO UNIVERSITY

MATH. SCIENCES RESEARCH INSTITUTE

NEW MEXICO STATE UNIV.

OREGON STATE UNIV.

\author{
STANFORD UNIVERSITY \\ UNIV. OF BRITISH COLUMBIA \\ UNIV. OF CALIFORNIA, BERKELEY \\ UNIV. OF CALIFORNIA, DAVIS \\ UNIV. OF CALIFORNIA, LOS ANGELES \\ UNIV. OF CALIFORNIA, RIVERSIDE \\ UNIV. OF CALIFORNIA, SAN DIEGO \\ UNIV. OF CALIF., SANTA BARBARA
}

\author{
Vyjayanthi Chari \\ Department of Mathematics \\ University of California \\ Riverside, CA 92521-0135 \\ chari@math.ucr.edu \\ Kefeng Liu \\ Department of Mathematics \\ University of California \\ Los Angeles, CA 90095-1555 \\ liu@math.ucla.edu \\ Jie Qing \\ Department of Mathematics \\ University of California \\ Santa Cruz, CA 95064 \\ qing@cats.ucsc.edu
}

These supporting institutions contribute to the cost of publication of this Journal, but they are not owners or publishers and have no responsibility for its contents or policies.

See inside back cover or msp.org/pjm for submission instructions.

The subscription price for 2013 is US \$400/year for the electronic version, and \$485/year for print and electronic.

Subscriptions, requests for back issues and changes of subscribers address should be sent to Pacific Journal of Mathematics, P.O. Box 4163, Berkeley, CA 94704-0163, U.S.A. The Pacific Journal of Mathematics is indexed by Mathematical Reviews, Zentralblatt MATH, PASCAL CNRS Index, Referativnyi Zhurnal, Current Mathematical Publications and the Science Citation Index.

The Pacific Journal of Mathematics (ISSN 0030-8730) at the University of California, c/o Department of Mathematics, 798 Evans Hall \#3840, Berkeley, CA 94720-3840, is published monthly except July and August. Periodical rate postage paid at Berkeley, CA 94704, and additional mailing offices. POSTMASTER: send address changes to Pacific Journal of Mathematics, P.O. Box 4163, Berkeley, CA 94704-0163.

PJM peer review and production are managed by EditFLOW ${ }^{\circledR}$ from Mathematical Sciences Publishers.

PUBLISHED BY

mathematical sciences publishers

nonprofit scientific publishing

http://msp.org/

(C) 2013 Mathematical Sciences Publishers 


\section{PACIFIC JOURNAL OF MATHEMATICS}

Volume $263 \quad$ No. $2 \quad$ June 2013

Realizations of $B C_{r}$-graded intersection matrix algebras with grading 257 subalgebras of type $B_{r}, r \geq 3$

SANDEEP BHARGAVA and YUN GAO

Stable flags, trivializations and regular connections

283

Elie COMPOINT and EDUARDo COREL

Elliptic aliquot cycles of fixed length

353

NATHAN JONES

Asymptotic $L^{4}$ norm of polynomials derived from characters

DANIEL J. KATZ

Degree-three spin Hurwitz numbers

JUNHO LEE

$\left(\mathbb{Z}_{2}\right)^{3}$-colorings and right-angled hyperbolic 3-manifolds

YOULIN LI and JiMING MA

Real closed separation theorems and applications to group algebras

Tim NETZER and ANDREAS THOM

Uniqueness theorem for ordinary differential equations with Hölder continuity

YifEI PAN, MEI WANG and Yu YAN

An analogue to the Witt identity

G. A. T. F. DA CostA and G. A. ZimmermanN

On the classification of stable solutions to biharmonic problems in large dimensions

Juncheng Wei, Xingwang Xu and Wen Yang 